\title{
Identification of a novel DNMT1 mutation in a Chinese patient with hereditary sensory and autonomic neuropathy type IE
}

Wenxia Zheng ${ }^{1 \dagger}$, Zhenxing Yan ${ }^{1 \dagger}$, Rongni He${ }^{1}$, Yaowei Huang ${ }^{2}$, Aiqun Lin' ${ }^{1}$ Wei Huang ${ }^{1}$, Yuying Su${ }^{1}$, Shaoyuan $\mathrm{Li}^{3}$, Victor Wei Zhang ${ }^{3,4}$ and Huifang $X_{i e}^{1 *}$

\begin{abstract}
Background: DNA methyltransferase 1 (EC 2.1.1.37), encoded by DNMT1 gene, is one of key enzymes in maintaining DNA methylation patterns of the human genome. It plays a crucial role in embryonic development, imprinting and genome stability, cell differentiation. The dysfunction of this group of enzymes can lead to a variety of human genetic disorders. Until now, mutations in DNMT1 have been found to be associated with two distinct phenotypes. Mutations in exon 20 of this gene leads to hereditary sensory and autonomic neuropathy type IE, and mutations in exon 21 cause autosomal dominant cerebellar ataxia, deafness and narcolepsy.

Case presentation: Here we report a novel DNMT1 mutation in a sporadic case of a Chinese patient with cerebellar ataxia, multiple motor and sensory neuropathy, hearing loss and psychiatric manifestations. Furthermore, we elucidated its pathogenic effect through molecular genetics studies and revealed that this defective DNMT1 function is responsible for the phenotypes in this individual.
\end{abstract}

Conclusion: Our findings expand the spectrum of DNMT1-related disorders and provide a good example of precision medicine through the combination of exome sequencing and clinical testing.

Keywords: DNMT1, HSAN1E, Exome sequencing

\section{Background}

DNA methyltransferase 1 (EC 2.1.1.37), encoded by DNMT1 gene, is one of key enzymes in maintaining DNA methylation patterns of the human genome. It plays a crucial role in embryonic development, imprinting and genome stability, cell differentiation $[1,2]$. The dysfunction of this group of enzymes can lead to a variety of human genetic disorders. Human DNMT1 consists of a large $\mathrm{N}$-terminal region harboring multiple conserved domains and a conserved C-terminal catalytic core. The large $\mathrm{N}$-terminal region is composed of the DMAP1-binding domain (DNA methyltransferase-associated protein 1), the proliferating cell nuclear antigen-binding domain (PBD), the replication focus targeting sequence (TS) 4

\footnotetext{
* Correspondence: xhffhx@126.com

tWenxia Zheng and Zhenxing Yan contributed equally to this work.

${ }^{1}$ Department of Neurology, Zhujiang Hospital, Southern Medical University, 253 Industrial Avenue Middle, Guangzhou City 510282, Guangdong Province, China

Full list of author information is available at the end of the article
}

domains, the CXXC domain, and two bromo-adjacent homology (BAH) domains [3]. Until now, all the mutations are found to be located in TS. TS is dispensable for the selective DNA methylation activity, but it can keep DNA from gaining access to the catalytic center and undergoing methylation by inserting into the catalytic pocket stably $[4,5]$. So far mutations in DNMT1 have been reported to cause two distinct neurological syndromes: hereditary sensory and autonomic neuropathy type IE with dementia and deafness (HSAN1E) related to mutations in exon 20 and autosomal dominant cerebellar ataxia, deafness and narcolepsy (ADCA-DN) with hearing loss and narcolepsy related to mutations in exon 21 [1].

\section{Case presentation}

A 38-year-old female from Guangdong province in China was admitted to Zhujiang Hospital, Southern Medical University in June 2016. At the age of 30, she developed progressive poor gait balance so that she 
frequently fell down when walking. At that time, she went to a local hospital for treatment, but diagnosis was not established. She was transferred to the Second Hospital Affiliated to Guangzhou Medical Hospital for hospitalization on December in 2010, where she was clinically diagnosed as cerebellar atrophy, Type 2 Diabetes and hyperlipemia. However, the treatments prescribed did not prevent disease worsening. In 2015, she presented a slowly progressing retardation. Within the year 2016, she began to suffer from bad-response, psychiatric manifestations, bilateral hearing loss and intermittent convulsion in her upper limb during sleeping, especially the right upper limb. With such complex symptoms, she was referred to our hospital. Her parents were not consanguinity, and no neurological disorders were found in her family members except herself.
Physical examination showed that she had mild mental retardation, apathy and spoke few words. Cranial nerves were normal except for symmetric bilateral sensory hearing loss. She did not cooperate with the neurological examination and sensibility could not be tested. Her muscle strength of bilateral upper limbs was normal but was decreased in lower limbs. Muscle tone was normal, but her right upper limb had abnormal involuntary movement. The patient had no pyramidal signs. Cerebellar function examination showed mild abnormalities on finger- to- nose, heel- to-knee and rapidly alternating pronation and supination of hands. Romberg test was negative.

Electrocardiogram investigation demonstrated sinus bradycardia (44/min on average). Nerve conduction studies revealed significant deceleration of motor conduction velocity in the right peroneal nerve $(25.9 \mathrm{~m} / \mathrm{s})$,

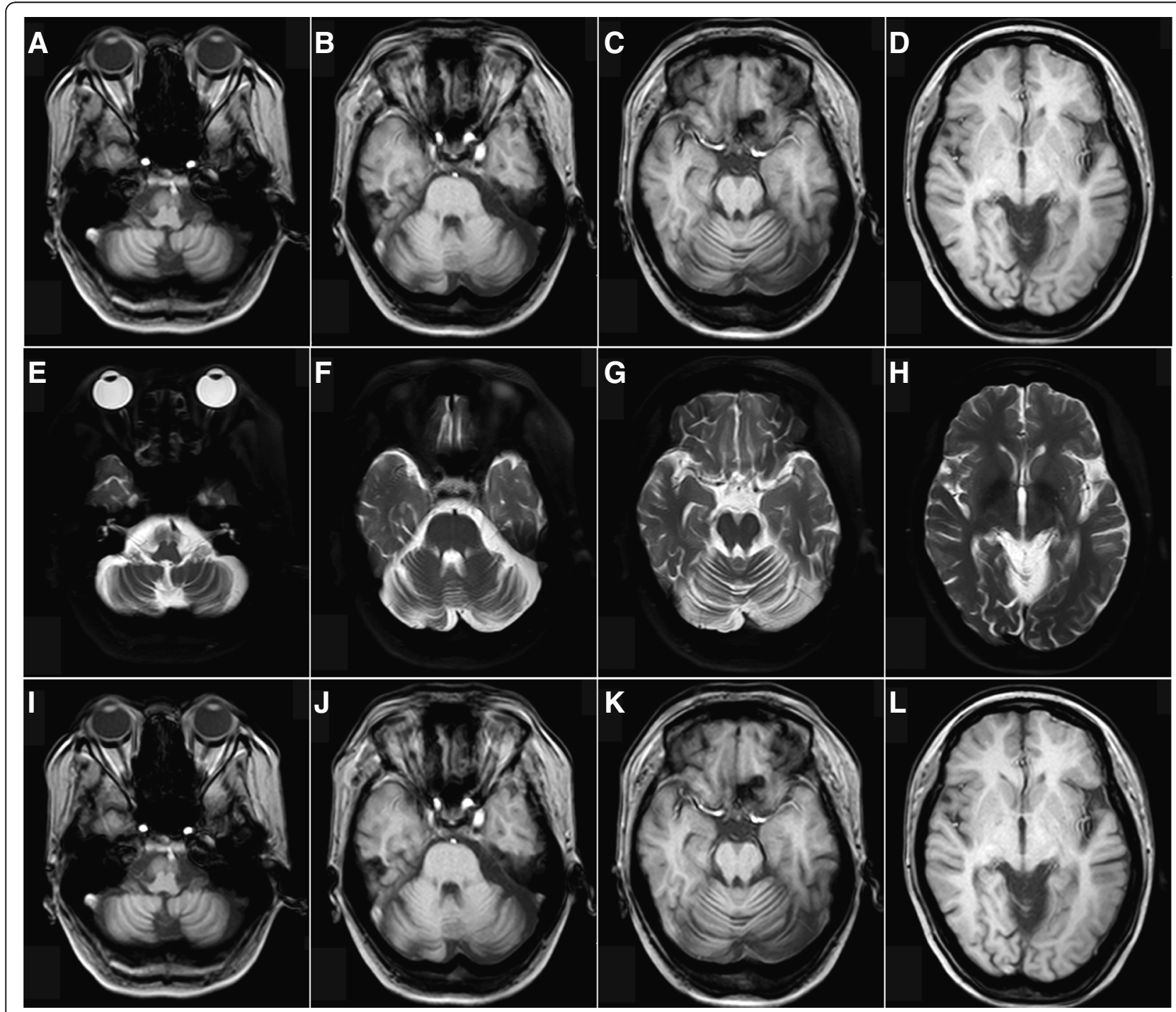

Fig. 1 Diffuse brain and cerebellar atrophy, especially the cerebellum. Sulcus inbilateral frontal, parietal, occipital lobe increases in width and depth (a-d: T1 weighted imaging, e-h: T2 weighted imaging, i-l: Fluid attenuated inversion recovery) 
right median nerve $(38.4 \mathrm{~m} / \mathrm{s})$, left ulnar nerve $(45.5 \mathrm{~m} / \mathrm{s})$, right ulnar nerve $(35.6 \mathrm{~m} / \mathrm{s})$ and the prolongation of $\mathrm{F}$ wave latency. The sensory nerve action potential could not be evoked in the bilateral peroneal nerve, sural nerve, and left median ulnar nerves. Electroencephalogram demonstrated mild to moderate abnormality and showed basic rhythms as $7.2-10 \mathrm{~Hz}$ and $20-40 \mu \mathrm{V}$ slow alpha activities with low amplitude and irregular wave. Few $14-25 \mathrm{~Hz}$ and $20-30 \mu \mathrm{V}$ beta waves sporadically emerged and more $4-7 \mathrm{~Hz}$ and $20-40 \mu \mathrm{V}$ theta waves presented sporadically or in short-term in each lead. Visual evoked potential showed the extending of bilateral latency of P100 (Left $=101.1 \mathrm{mS}$, right $=107.0 \mathrm{mS}$ ) and decreased amplitude (Left $=3.24 \mu \mathrm{V}$, right $=1.30 \mu \mathrm{V}$ ). Brainstem auditory evoked potential showed bilateral disappearance of III-I, V-I and V-III IPL. Brain MRI revealed diffuse cerebral and cerebellar atrophy (Fig. 1).

Exome sequencing [6] (as published) performed in AmCare Genomics Lab revealed a novel heterozygous missense variant, c $1618 \mathrm{~T}>\mathrm{A}$ (p. Y540N), in exon 20 of the DNMT1 (Fig. 2; Additional file 1).

\section{Discussion and conclusions}

We report a Chinese patient with suspected HSAN1E, which was finally confirmed by exome sequencing. HSAN1E is an autosomal dominant neural degenerative disorder related to the central and peripheral nervous systems characterized by sensory loss, deafness and dementia. Our patient appeared clinically normal until developing ataxic gait at hers 30s then abnormal behavioral presentations, following by hearing loss in hers 40s, It is different from the stereotypic progressive onset of hearing loss and sensory neuropathy at first and developed mental decline later. SPECT, PET image and autopsy studies in a Japanese kinship suggested the potential frontal lobe involved and abnormal behavioral presentations $[7,8]$. In contrast, a previous reported study about a large American family with 11 patients clinically examined but without behavioral presentations showed that a global process without selective frontal or subcortical involvement was indicated by brain autopsy of 3 affected patients, imaging studies and multiple neuropsychometric data [7]. It is worthy to noting that although our patient appeared with abnormal behavioral presentations and mental retardation observed by neurologist, diffuse cerebral and cerebellar atrophy instead of only frontal lobe involvement. Nerve conduction studies revealed sensory involvement. Therefore, her significant cerabellar atrophy and loss of deep sensation lead to atoxic gait. All these findings were consistent with HSAN1E but different from typical HSAN1E. Additionally, recent study further supports that the extent of the phenotypic spectrum in DNMT1-related disorders is much broader and more common than currently appreciated and estimated [9].

It is noteworthy that our patient has a novel variant $\mathrm{c}$ 1618 T > A (p.Y540N) in DNMT1 in exon 20. Using Sanger

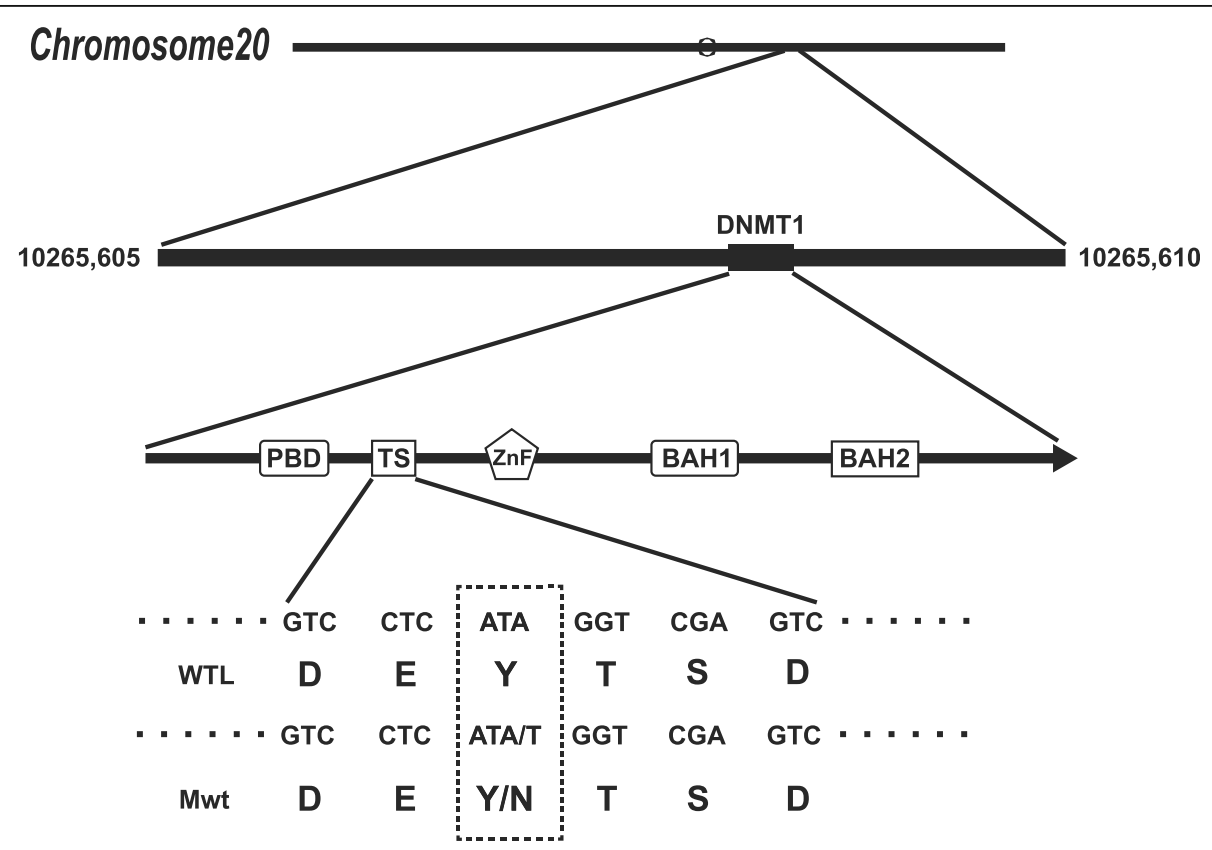

Fig. 2 Schematic representation of DNMT1 gene structure and its multiple domains in the N-terminal region. The boxes indicate PBD (the proliferating cell nuclear antigen-binding domain), TS (the replication focus targeting sequence), ZnF (zinc finger), BAH (bromo-adjacent homologydomain).The position of the mutation in the proband is indicated in the dotted box. The sequence data for mutation was deconvoluted to indicate a novel heterozygous missense variant. The tyrosine $(Y)$ residue at amino acid position 540 is replaced with asparagine amino acid residue. 
sequencing, we did not found the same variant in her mother. Unfortunately, we could not perform Sanger sequencing in her father since her father had already passed away at that time. However, although he did not appear any neurological manifestations until his death, we cannot make sure whether he carried the same mutation or not. Her siblings were not willing to accepting Sanger sequencing. But considering about her father and siblings without any neurological symptoms, we assume that her father should not have the variant. The identification heterozygous mutations in DNMT1 in patients have been published [2,9]. Therefore, we determined that c $1618 \mathrm{~T}>\mathrm{A}(\mathrm{p}$. Y $540 \mathrm{~N})$ was a pathogenic variant. So far, the mutation, c $1618 \mathrm{~T}>\mathrm{A}$ (p. Y540N), has not been discovered in our or other genetic database, neither published in clinical reports. Based on these results, we made a conclusion that the proband is likely to having a novel heterozygous pathogenic missense variant responsible for these symptoms.

In this study, we found a novel heterozygous missense variant, c $1618 \mathrm{~T}>\mathrm{A}$ (p. Y540N) in exon 20 of the DNMT1, which is associated with HSAN1E. Meanwhile, both clinical assessment and genetic tests of this patient are quite different from the typical HSAN1E. Our findings expand the spectrum of DNMT1-related disorders and provide a good example of precision medicine through the combination of exome sequencing and clinical testing.

\section{Additional file}

Additional file 1: The sequence data for novel mutation in DNMT1. The sequence data reveals a novel heterozygous missense variant, c 1618 T > A (p. Y540N), in exon 20 of the DNMT1. The tyrosine $(Y$ ) residue at amino acid position 540 is replaced with asparagine amino acid residue. (PDF 255 kb)

\section{Abbreviations \\ ADCA-DN: Autosomal dominant cerebellar ataxia, deafness and narcolepsy; $\mathrm{BAH}$ : Bromo-adjacent homology; DMAP1-binding domain: DNA methyltransferase-associated protein 1; DNMT1: DNA methyltransferase 1; HSAN1E: Hereditary sensory and autonomic neuropathy type IE with dementia and deafness; PBD: Proliferating cell nuclear antigen-binding do- main; TS: Replication focus targeting sequence}

\section{Acknowledgements}

The authors thank the family and patient for their participation to this study.

\section{Funding}

This work is Major Medical Collaboration and Innovation Program of Guangzhou Science Technology and Innovation Commission (grant number 201604020020) to WWZ.

\section{Availability of data and materials}

All data generated or analyzed during this study are included in the published article.

\section{Authors' contribution}

WZ and ZY drafted the manuscript. $\mathrm{RH}$ and $\mathrm{YH}$ collected the clinical data. AI,WH and YS participated in the care of the patient. SL and WWZ performed the gene panel sequencing and paper review. HX conceptualized and executed the case report. All authors read and approved the final manuscript.

Ethics approval and consent to participate

This case report was approved by the Ethics Board of Zhujiang Hospital, Southern Medical University.

\section{Consent for publication}

The mother of the patient and the patient herself provided signed informed consent for publication of this case report and any accompanying images.

\section{Competing interests}

The authors declare that they have no competing interests.

\section{Publisher's Note}

Springer Nature remains neutral with regard to jurisdictional claims in published maps and institutional affiliations.

\section{Author details}

'Department of Neurology, Zhujiang Hospital, Southern Medical University, 253 Industrial Avenue Middle, Guangzhou City 510282, Guangdong Province, China. ${ }^{2}$ Department of Neurology, Nanfang Hospital, Southern Medical University, 1838 Guangzhou Avenue North, Guangzhou, Guangzhou 510515, China. ${ }^{3}$ AmCare Genomics Lab, Guangzhou, Guangzhou 510320, China.

${ }^{4}$ Department of Molecular and Human Genetics, Baylor College of Medicine, Houston, TX 77030, USA.

Received: 29 June 2018 Accepted: 14 October 2018

Published online: 20 October 2018

\section{References}

1. Fox R, Ealing J, Murphy H, Gow DP, Gosal D. A novel DNMT1 mutation associated with early onset hereditary sensory and autonomic neuropathy, cataplexy, cerebellar atrophy, scleroderma, endocrinopathy, and common variable immune deficiency. J Peripher Nerv Syst. 2016;21(3):150-3.

2. Sun Z, Wu Y, Ordog T, Baheti S, Nie J, Duan X, Hojo K, Kocher JP, Dyck PJ, Klein CJ. Aberrant signature methylome by DNMT1 hot spot mutation in hereditary sensory and autonomic neuropathy 1E. EPIGENETICS-US. 2014; 9(8):1184-93.

3. Yuan J, Higuchi Y, Nagado T, Nozuma S, Nakamura T, Matsuura E, Hashiguchi A, Sakiyama Y, Yoshimura A, Takashima H. Novel mutation in the replication focus targeting sequence domain of DNMT1 causes hereditary sensory and autonomic neuropathy IE. J Peripher Nerv Syst. 2013;18(1):89-93.

4. Maresca A, Zaffagnini M, Caporali L, Carelli V, Zanna C. DNA methyltransferase 1 mutations and mitochondrial pathology: is mtDNA methylated? Front Genet. 2015;6:90

5. Garvilles RG, Hasegawa T, Kimura H, Sharif J, Muto M, Koseki H, Takahashi S, Suetake I, Tajima S. Dual functions of the RFTS domain of Dnmt1 in replication-coupled DNA methylation and in protection of the genome from aberrant methylation. PLoS One. 2015;10(9):e137509.

6. Barboza-Cerda MC, Wong $\sqcup$, Martinez-de-Villarreal LE, Zhang WW, Dector MA. A novel EBP c.224T>a mutation supports the existence of a malespecific disorder independent of CDPX2. Am J Med Genet A. 2014;164A(7): 1642-7.

7. Klein CJ, Bird T, Ertekin-Taner N, Lincoln S, Hjorth R, Wu Y, Kwok J, Mer G, Dyck PJ, Nicholson GA. DNMT1 mutation hot spot causes varied phenotypes of HSAN1 with dementia and hearing loss. Neurology. 2013; 80(9):824-8

8. Klein CJ, Botuyan MV, Wu Y, Ward CJ, Nicholson GA, Hammans S, Hojo K, Yamanishi H, Karpf AR, Wallace DC, et al. Mutations in DNMT1 cause hereditary sensory neuropathy with dementia and hearing loss. Nat Genet. 2011;43(6):595-600.

9. Baets J, Duan X, Wu Y, Smith G, Seeley WW, Mademan I, McGrath NM, Beadell NC, Khoury J, Botuyan MV, et al. Defects of mutant DNMT1 are linked to a spectrum of neurological disorders. Brain. 2015;138(Pt 4):845-61. 